\title{
Break-ups and the limits of encoding love
}

\author{
Bernie Hogan \\ Senior Research Fellow, Oxford Internet Institute \\ University of Oxford
}

\section{Citation}

Hogan, B (2018). Break-ups and the limits of encoding love. In Papacharissi, Z., ed. “Networked Selves and Love”. New York, NY: Routledge. Pp. 113-128.

\begin{abstract}
This essay explores how social network sites encoded relationships in ways that do not always fit the relationship as lived experience by individuals. This act of encoding is especially salient in acts of the relationship where there is a discrepancy between what is performed (love within the relationship) and what is exhibited (static states that signify the relationship). One critical case of this discrepancy is the break-up, as this process leaves static traces to persist online while individuals live separately. The essay uses examples from television media and psychology, particularly the concept of verbal overshadowing to emphasize the challenge in reconciling performed and exhibited relations. In doing so, this essay highlights that it is not only individual profiles and contributions that are curated on social media systems, but relationships as well. As a consequence, networked societies cannot contain aspects of human experience such as love which are relational and processoriented, but only signify these aspects and seek to steer them. The way they are steered is not necessarily in the interests of the individuals but the platforms. Designing for a break-up further implies considering how to undermine the pervasive logic of connectivity within the ideology of a network society.
\end{abstract}


"We say, "The wind is blowing," as if the wind were actually a thing at rest which, at a given point in time, begins to move and blow...This reduction of processes to static conditions, which we shall call "process-reduction" for short, appears self-explanatory to people who have grown up with such languages."

- Norbert Elias (As quoted in Emirbayer [1997])

\section{Introduction}

A relationship as experienced by people is a process. In order for it to be leveraged by computers, however, it must be turned into a static thing that signifies the state of that relationship. While we might suggest that such a conversion is a harmless and trivial matter, there are multiple instances when this conversion can create tension, unease, subversion or confusion. One clear example of this is in the case of the "break-up" whereby one or both parties wishes to cease their relationship or to alter it so that both people no longer interact with each other as if they are doing so jointly.

Break-ups are a compelling topic for thinking about the networked society as they are both very common and run counter to the logic of connectivity that powers much of network society. The logic of connectivity is that connections between individuals or individuals and entities that are real should be accounted for. It is thought that the more such connections are accounted for, the more effectively any algorithm can facilitate future connections (Hogan 2015), particularly for advertising purposes and enhanced user experience. A breakup signifies a dissolution of a link as two people change status from in a relationship to no longer in a relationship. While the ability to change a status, defriend another and even block someone is included within most social network sites, these choices are often implemented with little clarity. As will be shown below, the difficulty with breaking up 
online is that by their very nature, online profiles and the links between them do not cleanly map on to the lived experience of a relationship.

As Gershon noted in The Breakup 2.0 (2010), young adults face a dizzying array of strategies when breaking up, from blocking, to changing statuses, to "ghosting" (offering noresponse). These are in addition to the shifting norms on what is the appropriate medium within which to send the message. Beyond the evolving social norms of whether to Snapchat a break-up message or send by post is a far deeper matter of how network societies structure and control relations. Network societies thrive on connectivity as well as operationalization. The break-up is a "breaching moment" (Garfinkel, 1967) for the networked self. Breaching experiments occur when individuals intentionally violate expected norms or conventions in order to understand how such norms are enacted in everyday life. When two individuals undermine the codified norms of connectivity on a social network site, they lay can bare the ideologies of connectivity that are otherwise latent. One key insight in such a breaching is that a relationship is not a static codified object, nor will it ever be.

\section{The exhibited self and the exhibited relation}

In past work I have written of the exhibited self as distinct from the performative self (Hogan, 2010). The performative self is how the individual behaves for a specific audience. It is Goffman's idea that we can think of everyday life as a performance, like a stage play. The idea of the performative self has taken on a rich and varied life. Perhaps the biggest turning point is via Butler's notion of performativity. Situated between the linguistic use of performance (e.g., "I pronounce you husband and wife") and Goffman's notion of performance, Butler notes that we reproduce conventions and identities continuously as we enact them. We do not merely have a gender, but continuously perform it with gestures that signify our masculinity or femininity, our sexuality, our class and so forth.

This notion of the self as a performance has been disrupted by social media. These media, and particularly social network sites are the quintessential form of media for the networked society. On these sites, performances happen every time we click to like a status update, 
upload a photo or write a post. We signify elements of ourselves that reveal and reinforce norms and identities. However, these performances are not presented as is to our audiences. Instead, they are filtered through a complex series of transformations, many of which are typically hidden from the person doing the performing. For example, when someone posts a status update on Twitter (I.e. A "tweet"), Twitter acts as a mediator. It adds "metadata" such as the time the tweet was sent, the device, and the location of the device (inferred or precise; Twitter, 2017). The tweet is not merely a performance, it is an artefact that is curated by Twitter. The platform decides whether to promote this tweet, whether to remove it, who to send it to in email updates or "since you've been gone" lists of tweets. What was once a simple performative gesture (saying something in under 140 characters) has now become part of a networked exhibit.

The notion of an exhibit reinforces the idea the current performances are mediated. Twitter, Facebook, Snapchat, Tumblr, and so forth host this data. In the past decade, it has become clear that there is too much socially relevant information for any individual to consume in any given sitting. Consequently, mediators have become curators. They do not simply intervene between two parties but exert agency. The most direct form of agency is in how they determine the sort order for a series of posts. They also insert advertisements in lists in order to extract some of the user's attention. Curators hide unpleasant posts (with varying degrees of accuracy). This newfound centrality of platforms in social life helps to reinforce the notion of life as networked life and individuals as networked individuals (Rainie \& Wellman, 2012).

For platforms to work as curators between people requires more than an exhibited self, it requires an exhibited relation. Unlike exhibited relations, exhibited selves have now been extensively explored in the literature. For example, Zhao et al., (2013) describe how individuals enact a lowest-common denominator self of exhibited artefacts even though what constitutes the lowest common denominator shifts over time. People often delete old statuses or clean up their online personae. Vivienne and Burgess (2012) show how trans persons and their families carefully negotiate expected audiences for this exhibited self when making self-representing documentaries. Both Vitak and Kim (2014) and Marwick and boyd (2011) highlight how individuals consider potential "nightmare friends" or other potential threats when deciding whether to post a potentially controversial status. This is to 
say, not only is there an exhibited self, but that this self is something individuals understand as a part of networked life.

Despite the extensive work in computer-mediated communication on the exhibited self, there is much less work on the exhibited relation. Boyd was an early entrant in this space whereby she critiqued the stable notion of a friend. A 'friend' on MySpace could have meant any number of possible relations, from a true friend to a status symbol, a peer-pressure based acquaintance and more (boyd, 2006). This destabilization of the friendship implies that what is signified online as a relation does not adequately capture the notions of relations understood in everyday life. But why? I assert that it is not because the label stands for many things. This would imply that we could just use more labels. They might be unwieldy from a user-interface perspective but they would be sufficient. Instead, I assert that it is because relationships are processes and labels are things which, at most, represent states.

In past work, I noted that death is the ultimate arbiter between the exhibited self and the performed self (Hogan, 2010). When individuals die, their exhibited selves live on. The body does not contain a "kill switch" for the variety of media accounts held by third parties. Accounts go dormant, or in the case of the most sophisticated platforms, such as Facebook, a third party can notify Facebook that the person has passed and that their profile should be memorialized (Facebook, 2017). Whereas death is the ultimate arbiter of the distinction between the performed and exhibited self, the break-up (between friends or lovers) can be seen as an arbiter of the distinction between the performed relation as a dynamic process and the exhibited relation as a static object. Break-ups are typically initiated by one person in the relationship and continue until both parties understand that the break-up has occurred. Typically, one person will speak or write to the other person and signify that the relationship has ended or that they wish to end it. This is a performative gesture. To note, sometimes one of the parties will not agree that the relationship is over, which can complicate the process of breaking up but it does not nullify the break-up process.

While the break-up has then occurred in the minds (and hearts) of the two in the relationship, the online signifiers of that relationship persist. This includes labels, tagged photos, and past statuses. One must consider what do to with a publicly declared online 
status, whether to unfriend or block the other person on any variety of media and whether to engage in some form of impression management. That is, the inadequate mapping between the lived relation that is performed and the networked relation that is exhibited needs to be resolved somehow.

\section{What is a world of networks?}

A world of networks is a world of particulars and relationships between them. The particulars might be people, accounts, photos, or pieces of text. If we can define a boundary we can say that everything inside this boundary is a particular. In social network analysis, everything contained by a particular boundary is a node. These nodes link to each other in some fashion. The set of nodes and relations define a network. We can observe the networks of people tagging each other in Facebook photos (Lewis et al., 2008), of HAM radio operators signaling each other (Bernard, Killworth and Sailer, 1982), of countries trading (Wallerstein, 1974) and indeed in many other ways.

We can distinguish between network societies and network analysis. In social network analysis, this linking was done by the analyst who would suggest some sufficient criteria. For example, a research might indicate links between two people if they have sent any direct communication in the last six months. This is obviously an artificial boundary, but often a workable one. With these links in place, we can build up a social network and analyze it as such, describe its overall features and make claims about how the structure of relationships has some sort of consequence to the people who make up the network. This, however, is not a network society. This is a network analysis.

In a network analysis, we turn phenomena into particulars (i.e. nodes) and create sufficient conditions for linking at the point of analysis. This might be in the lab or in a software program. In a network society, we turn phenomena into particulars at the point of practice. This is to say, we enact networks rather than merely analyze them. Healy has referred to this as the performativity of networks (2015). This distinction is immensely important, for what is critical in a network society is to interrogate how objects are turned into nodes and processes turned into links. 
This transformation to nodes and links makes the world a more calculable place. This is the general product of modernity. It is where we turn danger into risk (Beck, 1992; Luhmann, 1993). Gods, ghosts and goblins as abstract dangers recede from view. In their place are the calculable risks we embed in futures markets, logistics and insurance brokers. When these objects of calculation represent social phenomena, they are done by platforms that make expectations about what sort of profile and what sort of relating can be accomplished by people on that platform.

The problem with the transition to a world of particulars comes when we try to codify processes as static objects, and worse, feed these objects back into the system. Processes are hard to identify as they are dynamic in nature. We cannot capture wind. We can capture wind-energy through turbines and air that is blowing through pressurized tanks, but we cannot capture the movement of air for it is a process, not a thing.

To consider the relationship, it is useful to contrast it with a role and a status. For this I lean on Merton's notion of the "role set" (1996). In Merton's parlance, a status is an ascribed label, such as teacher or mother. A role is a particular configuration of social structure to accomplish some end. Roles never exist in isolation but as parts of role sets. Mothers have children, teachers have pupils. It is the structural arrangement of relationships that defines the role set. One can play a role, such as a nurse, by being nurse-like in their interactions with doctors and patients. If one is not supposed to play this role (I.e. They do not have the status of a nurse), then trouble might ensue for they do not have the status that signifies or legitimates that role.

A relationship is a specific process of interaction between two people. When a specific nurse deals with a specific patient this is a relationship. The patient has their own needs for care, their own history with the nurse. The two have a rapport shared among two mutually acknowledging persons. Unlike a role, a relationship is a dynamic process of continuouslynegotiated entrainment. Friendships come and go (Suitor and Keaton,1997; Antonucci, 1986). Our respect and affection for our family members waxes and wanes, often in concert with family drama (Wellman and Wortley, 1989) and differences in political opinions. The specific structuring of statuses within the family unit are proscribed by culture, such as "first 
cousin once removed". Such a status does not necessitate a specific relationship that would happen between cousins. They may be friends, enemies or strangers, and still be cousins.

The problem for network society herein is that relationships cannot be easily codified except when filtered through the roles that set up social structure and the statuses that are used to codify roles. We think of marriage as a relationship, yet it is not, at least not directly. In Mertonian terms, a marriage is a role set. Speak to many couples and it is clear that between them, there are differing levels of engagement, marital satisfaction and feelings of attachment (Norton, Baptist \& Hogan, 2017). The couples, by necessity, have different lives and experiences and thus different relationships between them. Consider this distinction in the subtleties of the definition of marriage: "the legally or formally recognized union of a man and a woman (or, in some jurisdictions, two people of the same sex) as partners in a relationship" ("Marriage", n.d.). This definition asserts that the relationship is pre-existing and presumably on-going. Marriage is not how the relationship starts. Rather, marriage is a formal recognition as a union. The term "husband" is a status. The way a husband relates to his partner, to the courts, and to family members is a role. The actual way the person behaves towards and feels about his husband or wife is his part of the relationship.

\section{Love and social media}

One of the most powerful and yet ambiguous processes in the world is love. A break-up neither eliminates love or happens because we fall out of love, yet the two are inextricably linked. As Appignanesi notes:

"Love is deeply private and particularly in its passionate form oft-ungovernable, while marriage is an institution, championed by regulatory states in the name of an ordered society. The contradiction between the two can produce a deep malaise - from which longterm and public cohabitation, that intermediate arrangement, is hardly free" (2011:111).

Love is not a static condition. It is a dynamic process whose qualities are known to many yet remain elusive to study. It is related to attachment, to fidelity, to rituals and to the life course. Some societies privilege love in relationships and some try to contain love in favour 
of more pragmatic relationships. No society denies there is love between a mother and child and few societies deny that courtship involves affection, even if it is accomplished within politically or culturally arranged marriages (Shorter, 1977).

Instead of exploring the relationship between love and the network society directly, we can create boundary conditions around the codification of love by considering how relationships are handled in social network sites, as exemplars of this networking logic. Herein, it is important to note that people are not the particulars, accounts are. People can have multiple accounts and use pseudonyms (Hogan, 2012xx). But each account has a set of profile data and a set of links to others. In this sense, the relations that people have with each other are not links in the way Facebook encodes them, or the way an analyst encodes them. Our lived experience only partially matches the technological encoding of our lived experience.

The mission of social networks, enacted through coders, designers, user experience researchers, security professionals and the bureaucracy that props up and facilitates their activities, is to encompass ever more of lived experience in the coded world. Consider Facebook's two most recent mission statements. Up until 2017 their motto was "To give people the power to share and make the world more open and connected." This has recently changed to "Give people the power to build community and bring the world closer together." (Newton, 2017)

The first motto noted that openness and connectivity were self-evident goods. They were ends in themselves and Facebook was to make the world more open. With upwards of two billion users, this ambition is hardly an overreach. While openness has been excised from the recent mission statement, what remains is the conception of Facebook as mediating "the world" and in doing so, aspiring to create some positive outcome. In this case, both community and bringing the world closer together are seen as self-evident virtues. Yet, in bringing the world closer, it must first encode the world in the eyes of Facebook as well as appeal to the shareholders, employees and advertisers who pay for this closeness.

Some things are more easily encoded than others. We can think of lossless coding for audio and photos, or screen resolutions so high that the human eye cannot distinguish distinct 
pixels. But what about encoding more abstract concepts like love or friendship? Most people would positively acknowledge the ontological status of both friendship and love. Most people believe love is, indeed, real. Nevertheless, love represents a considerable challenge for encoding. This is for at least three reasons:

1. Love is ambiguous. Does love represent infatuation? Affection? Care? A theory from antiquity proposed six distinct love styles (Eros, Storge, Pragma, Ludus, Agape and Mania). These styles apply to many kinds of love beyond pair-bonding, yet all are plausibly seen within couples. Recent work has shown that differing styles have differing relationship outcomes (Vedes et al., 2016).

2. Love is asymmetric. Early love has been termed limerence, an intense phase of infatuation that can border on obsession. Tennov has explored the life cycle of limerence, showing that it is temporary and often leads to stable romantic love, but that it does so to a different extent in different people (1999). Limerence, when unreciprocated, can produce intense feelings of heartache bordering on illness. The unrequited lover would not doubt the strength of their affection, only its feasibility.

3. Love is dynamic. In some of the oldest living couples, love persists in a variety of forms. Yet, feelings recognizable as love do wax and wane in both enduring couples and those who eventually break up (McNulty, Wenner, \& Fisher, 2016). While we might say that being out of love predicts relationship dissolution, it does not guarantee it. The love shared between even the happiest couples varies in its intensity over time.

Ambiguity, asymmetry and dynamism suggest that love is more like a process than an entity. Love is not a thing, it is a mode of relating. A network society cannot therefore encode love any more than it can code a relationship; it can only signify it and code the signification process. This signification tends to be done through a mix of categorical statuses and signals about the relationships between users - do the users click on each other's statuses on Facebook, thereby contributing to their increased prioritization in the newsfeed? Do they send each other the most snaps on Snapchat, thereby prompting Snapchat to show little emojis next to the other person's name? 
Broadly speaking, these signals are meant to stand in for love. Platforms substitute in the signals we can measure (such as promptness) for more abstract, contextual or relational signals that are more difficult to measure. With every action, every click, like, or tag, the individual is sending signals about their relationships that can then be used to update some strength of connections. Often this is done as part of a feedback loop where new relationships quickly entrain. Algorithms do not represent love, but steer it. Suddenly one lover is all over the other's feeds and limerence is not merely about repeatedly thinking of the other lover, but seeing them as well. Notably, this virtuous cycle of paying attention and then seeing one's lover appear at the top of a feed also applies for suggested purchases. When one purchases something on an online retailer, related purchases seem to follow the user around the web. With curated feeds and advertisements, a feedback loop of presentation and interest ensnares the user, steering attention towards both loved ones and products for sale.

Encoded data from platforms can steer a relationship to become more intense. This data can also predict the dissolution of a relationship. Backstrom \& Kleinberg (2014) have noted a significant relationship between the social networks of individuals on Facebook and their likelihood of breaking up. Other members of the Facebook data team have noted that when someone changes their relationship status to single from being in a relationship, Facebook activity from the other party in the relationship spikes, presumably as they solicit social support and engage in impression management (Friggeri, 2014).

On social media platforms such as Facebook and Twitter, the network does not emerge from interaction so much as presupposes the interaction. In this sense, platforms do more than steer relationships, but act as gatekeepers of access for these relationships as well. We add friends and then we see their content. Thus, media come to define the parameters for what is possible and what is available in terms of social connections. For example, on Facebook, one cannot tag a person in a photo who is not their friend. On Twitter, one typically cannot send a direct message to an account if that account does not follow the user (though this is now configurable). These rules are set up to filter unwanted access as well as encourage people to denote specific links between individuals. A networked logic does not simply emerge from the interaction on a platform, but from the design of the platform itself. 
To suggest that the world is networked does not merely mean that there are connections, but also that there are forms and protocols that permit specific kinds of connections. These protocols are currently set up and enacted by third parties who steer attention while they curate social signals. As these protocols cover more forms of social life, they require new kinds of behaviours and spark new anxieties. As stated above, the distinction between the lived relation and the static code can frustrate some dimensions of human behaviour. This has been felt in popular culture as much as in academic work. In fact, free from the demands of empirical work, popular culture can exaggerate these anxieties in ways that highlight some of the present challenges for the network society. In the case of handling the distinction between the exhibited status and the relation as process, the show Black Mirror raises important and compelling questions.

\section{Black Mirror and the fragmented digital}

The near future dystopian show, Black Mirror, offers myriad cases of break-ups gone awry. Two in particular stand out for their poignancy. The first is a season two episode called The Entire History of You (Armstrong \& Welsh, 2011), which is a meditation on how to deal with loss when everything can be remembered. The second, White Christmas (Brooker \& Tibbetts, 2014), is a meditation on how to deal with loss when everything is mediated. Since Black Mirror is a show about near-future dystopias, the show tends to include some novel technological features. In both break-up episodes, this centers around the use of digitally enhanced eyes. In The Entire History of You, the eyes act as a sort of camera that can record and store everything using a small technology called "The Grain". It shows up in security checks and people converse while replaying old clips. In White Christmas, we observe a world where the eyes serve as a sort of augmented reality, but the user does not have dominion. People can literally "block" each other. A blocked person is viewed as a static silhouette whose voice cannot be heard. The person doing the blocking becomes a ghost to those blocked. When a person in the show is revealed (and convicted) as a sex offender, their punishment is not prison but the total blocking of all people, who now exist as a sea of static figures. The sex offender is reciprocally shown as a red silhouette to everyone else. 
Both shows use visual technology as a narrative device that "virtualizes" the physical world; everything is now encoded and mediated. This sounds futuristic, but if we move beyond the need for digitally enhanced eyes that capture what we see, both stories are squarely embedded in the present.

The Entire History of You is perhaps the episode that is the closest to our current epoch. We can already set up cameras and record most of our daily lives. Some individuals already wear such cameras, most notably cyborg pioneer Steve Mann (Mann \& Niedzviecki, 2001) and "eyeborg" Rob Spence who lost one eye years ago and replaced it with a camera (Time, 2009). As Google Glass has shown, the norms around such recording are still unclear (Hong, 2013).

In The Entire History of You, the protagonist is concerned that his wife is having an affair. Checking the recordings from his wife, these suspicions become increasingly plausible. Further, she has deleted a key sequence of memories that occurred roughly nine months before their son is born. The audience is aware by this time that the protagonist is probably not the father. The episode then watches as the drama unfolds between these two characters.

The technologies for such a narrative almost exist today. By default, most texting applications store communication indefinitely. In some cases, we can turn on some sort of erasing on one end (for example Apple iMessage can be set to delete messages older than 30 days). Yet, if it is not built into the platform, there is no guarantee that both parties will delete the message. Snapchat is premised on the idea of self-destructing data. In fact, the Snapchat's creators went further in suggesting that sending pictures to a new romantic interest was what motivated the creation of the app in the first place (Crook \& Escher, 2015). Other programs such as Telegram and Whatsapp now include the ability to send photos that disappear after 30 days.

What happens to the artefacts of the other person after the break-up? They are currently linked to an account, not a person. The account is where deletion occurs and deletion is an active process that requires the user to actively remove content. That is to say, social network sites are typically not designed with a notion of decay in mind. Once a friend, 
always a friend. As a personal note, I can only recall Tumblr and LiveJournal as indicating that my account would expire due to a lack of activity. Facebook, Twitter and Linkedln provide no such feature. There are no affordances for how long it has been since last contact, or how to play 'catch up' on an account's activities since the last time the person viewed their friend. Here we can see how the technology in The Entire History of You that stores one's communications and senses are a mere extension of the digitization of interactions experienced in everyday life today.

In the case of both Black Mirror episodes, the ocular technology has become domesticated and institutionalized. Domesticated because we are show everyday practices that include such technologies as well as the subversion of norms. In White Christmas the protagonist sets up a shady business to help an awkward man on a date (watching through the awkward man's eyes). When the protagonist ends up witnessing a murder, this is seen as the subversion of acceptable norms. The ocular technology is institutionalized because when the protagonist is punished it is through the eye and ear pieces, by law. Similarly, in The Entire History of You the protagonist goes through customs only to have everything he saw on his trip played back by the customs officer. The drama from the show comes not from the technology of the eyes, but what is encoded, who gets to see it and when.

Part of the terror that emerges from these shows is not the ubiquity of the technology. It is dramatic tension set up via the uncanny valley in which the technology settles. Relationships are not absolute and binary. The line between taking interest in an ex-lover out of general welfare, appealing to an unhappy lover who is disinterested in talking and cyberstalking as an offense can be unclear for some. For those who are in aggressive pursuit of a disinterested lover, cyberstalking "does not fundamentally differ from traditional, proximal stalking" (Sheridan \& Grant, 2007; p. 627). Yet, what has frustrated efforts to provide accurate estimates for cyberstalking is that the asymmetric gaze of viewing others' online presences is an encouraged behavior for most social media (Reyns, Henson \& Fisher, 2011). This is even evident even in the first definition of Social Network Sites by boyd and Ellison (2007). Their third criteria to define social network sites is that the user may traverse profiles. One here is not applying a symmetric gaze, where the viewer looks and the viewed can now perceive the viewer. Instead, it is an asymmetric gaze where the viewer looks at the profile and the viewed profile owner is not informed, or not told specifically. To note, 
within the current literature it is established that the lack of a victim's awareness of a specific event of cyberstalking does not legitimize or excuse the behavior.

Gershon (2010) among others notes how students wrestle with the temptations to view these old photos and living profiles as a routine part of the break-up experience. While jilted lovers might have always pined over old photos, what is novel is that they can now pine over an endlessly new stream of content. It is not a far step to think of them pining over high-fidelity life streams, endlessly negotiating the virtues of deleting and forgetting alongside the temptation to relive old memories, trapped as viewers of their own exhibitions.

The notion that one either has a memory, locked in and repayable, or does not, is foreign and upsetting. Our memories are fragile. Yet, the superimposition of digital technologies on top of everyday relationships requires us to encode our representations and associate them with profiles so that we may link to others. This is the central dilemma of the protagonist in The Entire History of You: remove The Grain and everything that comes with it, but finally excise the painful memories, or keep The Grain and live with the temptation to constantly revisit video clips that show his life to be a lie. There is no therapy, no new relationship and no trajectory that appears to be able to resolve this dilemma. It is instead as binary as the bits used to encode this data.

Benjamin (1967) noted that with the advent of the camera, for the first time we come to sympathize with a fixed view. There is much absent on stage left that we never see and must infer, regardless of the fidelity of what is there in front of the screen. This, Walther notes (2007) is part of the experience of the online world as "hyper personal". In a retort to earlier thinkers who besmirched the Internet as cold and impersonal, Walther noted that we tend to want to engage with full realized personas. We want to understand motivations and desires. Where these cues of history and emotion are absent, our brain will fill in the details. No amount of life streaming will undermine this cold fact. When we encode our relations as exhibitions, we necessarily give something up.

In a telling experiment in a completely perpendicular field, wine-tasting, we learn something that we can bring back to this essay. In a between-subjects experiment, half the wine tasters 
were asked to describe the wine in words and the other half were told to remember the taste as is. In a return visit to the lab, those who had described the wine (with the traditional adjectives like floral and chocolate) were actually worse at re-identifying the wine. Melcher and Schooler (1996) described this phenomenon as verbal overshadowing. They offer the caveat that it was only untrained wine drinkers who were worse at reidentifying the wines. To note, the trained wine drinkers were not better when writing down words; they simply were not worse than the trained drinkers who remembered the wine as an experience. The same phenomenon can happen with visual memories as well (Schooler and Engstler-Schooler, 1990). Our attempts to encode our experiences can interfere with our understanding of them, for we are bodies of lived experience and our memories encode as such. Our attempts to digitize, verbalize and otherwise capture the moment in order to make it networked and available can also interfere with the original memory. In seeking to capture and, in some respects, simplify our relationships to specific labels available on social network sites, we can make verbal overshadowing part of everyday life.

While some break-ups are definitive and absolute, many more are tumultuous and uncertain. The relationship may wither, people may become withdrawn, conflict may be resolved...for a time. In this context, there is something especially insidious about the notion that we can simply 'change our status'. It creates the illusion that our minds work as aggregators of static objects like computers do. It suggests that we are capable of such binarism. Yet, as was indicated here, our selves and our relationships are dynamic processes. We are co-constituted by our various entrainments with others. The performative gesture of breaking-up is meant to reconfigure the status between two individuals. By saying "I'm breaking up with you" we are really saying, we cannot use the statuses we used before. But as noted above, statuses are labels, not relationships.

Slotter et al., (2009) shows that clarity about one's self-concept has a significant effect on post-break-up distress. In this research, those who were less sure of themselves after a breakup felt more distressed. The markers online that persist in reminding someone of their links to another person can bring back notions of the relationship and thus again destabilize the self as one transitions to this newer sense of self after the break-up. This is reinforced by Lewandowski et al., (2006) who demonstrated that the remembrance of a past 
relationship was associated with a smaller self-concept than a control group. In both cases, we observe how the self itself ebbs and flows with signifiers of the external world. One's identity markers, such as a name or face, may remain relatively stable. Yet the phenomenon they represent (the self) is fragile and subject to variation based on external stimuli. By extension, networked selves are cultivated by interactions with particular other identities. The relationships that are signified by these identities are in flux and only ever approximately signified by status such as 'is friend' or 'in a relationship'.

These studies suggest that the technologies designed to keep us "open and connected" (as was Facebook's recent mission statement), do little to manage what happens when we want to close off and disconnect from others. Instead of acting as coherent gatekeepers, the technologies typically work in an all or nothing fashion. We know that online activity spikes after an individual is broken up with (Friggeri, 2014), but we also know that there is no pleasant way to deal with the persistent and enmeshed markers of a past life. No one will expect to see a room full of photos of a wife and her ex-husband, yet, Facebook will still insensitively bring up old memories, even "friendaversaries".

What does this portend for life and love in the networked world? Is love even possible anymore? Surveys and interviews asking if people can find love will still inevitably return in the affirmative. Love is one of the strongest and most important dimensions of the human experience, regardless (or perhaps because) of its ineffable qualities. Yet, in contrast to this mysterious force, the networked world is one of operationalization and calculability. The networked world operates on its own "big data" logic (boyd and Crawford, 2012). Part of this logic is to encourage individuals to put as much online as possible in order to optimize online algorithms (Hogan, 2015). With increased information comes increased calculability. Advertisements get more relevant, lists are ranked in a more personalized order, media become more adept at circumscribing the self. Yet, as noted above, this ideology necessarily involves the alchemy of turning processes into things, and things into encoded things. Love, like the wind, is a process and not a thing. The networked world can facilitate it for the lonely or provide cruel and partial reminders when it leaves. But the networked world cannot contain love any more than a windmill's fins can contain the wind. 
By circumscribing these limits, it is not my intention to undermine the networked world.

Such a task would be as foolhardy as it was unrealistic. It is instead to provide a reminder of limits of encoding, regardless of the media. In doing so, it should enable individuals to step back from a triumphant discourse of digital connectivity. If we design for connection, we must also design for disconnection, and understand that both will only approximate the experience. As a fortuitous example of this hubris in action, while writing this conclusion Tumblr alerted my phone that "You'll never know love until you see this post". No doubt, the post and likely the timing of the alert, were selected algorithmically. If social media cannot contain love, at least it can trivialize it. Yet, by appreciating the difference between processes and things, exhibitions and performances, we may be more humble in the face of one of humanities most painful and frustrating experiences, the break-up. We might also be humbled to the reason we risk such a tumultuous experience in the first place: to engage in the ineffably joyous process of love.

\section{References}

Antonucci, T. (1986). Measuring Social Support Networks: Hierarchical Mapping Technique. Generations, 10, 10-12.

Appignanesi, L. (2011). All About Love: Anatomy of an Unruly Emotion. New York, NY: W. W. Norton \& Company.

Armstrong, J. (Writer) \& Welsh, B. (Director). (2011). The Entire History of You [Season 1, episode 3]. In Brooker, C. \& Jones, A. (Executive Producers), Black Mirror. London, UK: Channel 4.

Backstrom, L., \& Kleinberg, J. (2014). Romantic Partnerships and the Dispersion of Social Ties: A Network Analysis of Relationship Status on Facebook. In Proceedings of the 17th ACM conference on Computer Supported Cooperative Work. Baltimore, MD.

Beck, U. (1992). Risk society: Towards a new modernity. Thousand Oaks, CA: Sage.

Benjamin, W. (1967). The work of art in the age of mechanical reproduction. In Illuminations (pp. 217-251). New York: Schocken Books.

Bernard, H. R., Killworth, P. D., \& Sailer, L. (1982). Informant accuracy in social-network data V. An experimental attempt to predict actual communication from recall data. Social Science Research, 11(1), 30-66. 
boyd, D., \& Crawford, K. (2012). Critical Questions for Big Data. Information, Communication \& Society, 15(5), 662-679.

boyd, danah, \& Ellison, N. (2007). Social Network Sites: Definition, History, and Scholarship. Journal of Computer Mediated Communication, 13(1), 210-230.

boyd, danah. (2006). Friends, Friendsters and Top 8: Writing Community into being on social network sites. First Monday, 11(12).

Brooker, C. (Writer) \& Tibbetts, C. (Director). (2014). White Christmas [Season 2, episode 4]. In Brooker, C. \& Jones, A. (Executive producers), Black Mirror. London, UK: Channel 4.

Crook, J \& Escher, A. (2015, October 15). A brief history of Snapchat. TechCrunch. Retrieved from https://techcrunch.com/gallery/a-brief-history-of-snapchat/

Emirbayer, M. (1997). Manifesto for a relational sociology. American Journal of Sociology, 103(2), 281-317.

Facebook. (2017). Memorialized Accounts. Retrieved from https://www.facebook.com/help/1506822589577997/

Friggeri, A. (2014) When Love Goes Awry. Facebook Data Science. Retrieved online from https://www.facebook.com/notes/facebook-data-science/when-love-goesawry/10152066701893859/

Garfinkel, H. (1967). Studies in Ethnomethodology. Englewood Cliffs, NJ: Prentice-Hall.

Gershon, I. (2010). The Breakup 2.0: Disconnecting over New Media. Ithaca, New York: Cornell University Press.

Healy, K. (2015). The performativity of networks. European Journal of Sociology, 56(2), 175205.

Hogan, B. (2010). The Presentation of Self in the Age of Social Media: Distinguishing Performances and Exhibitions Online. Bulletin of Science, Technology \& Society, 30(6), 377-386.

Hogan, B. (2015). From Invisible Algorithms to Interactive Affordances: Data After the Ideology of Machine Learning. In Roles, Trust, and Reputation in Social Media Knowledge Markets (pp. 103-117). Springer.

Hong, J. (2013). Considering Privacy Issues in the Context of Google Glass. Communications of the ACM, 56(11), 10-11.

Lewis, K., Kaufman, J., Gonzalez, M., Wimmer, A., \& Christakis, N. (2008). Tastes, ties, and time: A new social network dataset using Facebook. com. Social Networks, 30(4), 330342. 
Luhmann, N. (1993). Risk: a sociological theory. New York: Walter de Gruyter.

Mann, S., \& Niedzviecki, H. (2001). Cyborg: Digital destiny and human possibility in the age of the wearable computer. Toronto, Canada: Doubleday Canada.

Marriage. (n.d.). In Oxford English dictionary. Retrieved from https://en.oxforddictionaries.com/definition/marriage

Marwick, A. E., \& Boyd, D. (2011). I tweet honestly, I tweet passionately: Twitter users, context collapse, and the imagined audience. New Media \& Society, 13(1), 114-133. https://doi.org/10.1177/1461444810365313

McNulty, J. K., Wenner, C. A., \& Fisher, T. D. (2016). Longitudinal Associations Among Relationship Satisfaction, Sexual Satisfaction, and Frequency of Sex in Early Marriage. Archives of Sexual Behavior, 45(1), 85-97.

Melcher, J. M., \& Schooler, J. W. (1996). The Misremembrance of Wines Past: Verbal and Perceptual Expertise Differentially Mediate Verbal Overshadowing of Taste Memory. Journal of Memory and Language, 35(2), 231-245.

Merton, R. K. (1996). The Role-Set. In P. Sztompka (Ed.), On Social Structure and Science (pp. 113-122). Chicago: University of Chicago Press.

Newton (2017, February 16). Facebook just changed its mission, because the old one was broken. The Verge. Retrieved from https://www.theverge.com/2017/2/16/14642164/facebook-mark-zuckerberg-lettermission-statement

Norton, A. M., Baptist, J., \& Hogan, B. (2017). Computer-Mediated Communication in Intimate Relationships: Associations of Boundary Crossing, Intrusion, Relationship Satisfaction, and Partner Responsiveness. Journal of Marital and Family Therapy, 1-18.

Rainie, L., \& Wellman, B. (2012). Networked: The New Social Operating System. Cambridge, MA: MIT Press.

Reyns, B. W., Henson, B., \& Fisher, B. S. (2011). Being Pursued Online: Applying Cyberlifestyle-Routine Activities Theory to Cyberstalking Victimization. Criminal Justice and Behavior, 38(11), 1149-1169.

Schooler, J. W., \& Engstler-Schooler, T. Y. (1990). Verbal overshadowing of visual memories: Some things are better left unsaid. Cognitive Psychology, 22(1), 36-71.

Sheridan, L. P., \& Grant, T. (2007). Is cyberstalking different? Psychology, Crime \& Law, 13(6), 627-640.

Shorter, E. (1977). The Making of the Modern Family. Basic Books. 
Suitor, J., \& Keeton, S. (1997). Once a friend, always a friend? Effects of homophily on women's support networks across a decade. Social Networks, 19, 51-62.

Tennov, D. (1999). Love and Limerence. London, UK: Scarborough House.

Time. (2009) The Eyeborg. The best 50 inventions of 2009. Retrieved from http://content.time.com/time/specials/packages/article/0,28804,19340271934003 1 $\underline{933989,00 . h t m l}$

Twitter (2017). Tweet Data Dictionaries. Retrieved online from https://developer.twitter.com/en/docs/tweets/data-dictionary/overview/tweet-object

Vedes, A., Hilpert, P., Nussbeck, F. W., Randall, A. K., Bodenmann, G., \& Lind, W. R. (2016). Love styles, coping, and relationship satisfaction: A dyadic approach. Personal Relationships, 23(1), 84-97.

Vitak, J., \& Kim, J. (2014). You can't block people offline: examining how Facebook's affordances shape the disclosure process. Proceedings of the 17th ACM Conference on Computer Supported Cooperative Work, 461-474.

Vivienne, S., \& Burgess, J. (2012). The Digital Storyteller's Stage: Queer Everyday Activists Negotiating Privacy and Publicness. Journal of Broadcasting \& Electronic Media, 56(3), 362-377. https://doi.org/10.1080/08838151.2012.705194

Wallerstein, I. (1974). The modern world system: Capitalist agriculture and the origins of the European world economy in the sixteenth century. New York, NY: Academic Press.

Walther, J. B. (2007). Selective self-presentation in computer-mediated communication: Hyperpersonal dimensions of technology, language, and cognition. Computers in Human Behavior, 23, 2538-2557.

Wellman, B., \& Wortley, S. (1989). Brothers' keepers: Situating kinship relations in broader networks of social support. Sociological Perspectives, 32(3), 273-306.

Zhao, X., Salehi, N., \& Naranjit, S. (2013). The many faces of Facebook: Experiencing social media as performance, exhibition, and personal archive. Proceedings of the SIGCHI Conference on Human Factors in Computing Systems. [CHI '13]. Paris, France. 